\title{
Dynamics of Gold Nanoparticles at the Solid: Liquid Interface Studied by Liquid-Phase Electron Microscopy
}

\author{
$\underline{\text { Elisa Cepeda-Pérez }}^{1}$ and Niels de Jonge ${ }^{1,2}$
}

1. INM - Leibniz Institute for New Materials, Saarbrücken, Germany.

2. Department of Physics, University of Saarland, Saarbrücken, Germany.

The dynamics of nano-sized objects at solid:liquid interfaces plays a key role in a variety of relevant phenomena such as friction, self-assembly, surface-based technologies related to catalysis and molecular targeting among others [1]. Therefore, the study of nanoparticle dynamics at solid:liquid interfaces has been the subject of extensive research. However, a complete model of surface transport at interfaces is missing and certain aspects of the interactions between nanoparticles are still not well understood [2].

The mechanisms of diffusion of colloidal nanoparticles at a solid:liquid interface are known to be related to the chemical and physical characteristics of the colloid [3]. For metallic nanoparticles dispersed in water, the dynamical movements in bulk correspond to Brownian motion and the stability of the system is ruled by electrostatic interactions where the key parameters are: the ionic strength of the solution, the surface potential of the nanoparticles and the elemental nature of the metallic nanoparticle. But under confinement conditions near a solid:liquid interface the dynamical behavior becomes anomalous, it cannot be described in terms of Brownian motion [4] and a variety of phenomena such as the intermittent "hopping" and oriented assembly of the nanoparticles have been observed $[5,6]$.

Here, we used liquid-phase scanning transmission electron microscopy (STEM) to analyze the dynamics of individual gold nanoparticles (AuNPs) in solution at the solid: liquid interface during a self-assembly process triggered by the electron beam. Our experimental setup consisted of two silicon microchips, each containing a $50 \mathrm{~nm}$-thick electron transparent silicon nitride ( $\mathrm{SiN}$ ) window. The microchips were placed one on top of the other so that a liquid enclosure was formed between both windows. To ensure a defined distance between the chips, one of the silicon microchips included a spacer of $0.5 \mu \mathrm{m}$ height. On one of the microchips, we deposited by direct dripping $0.3 \mu \mathrm{l}$ of a colloidal solution containing $20 \mathrm{~nm}$-diameter AuNPs. The microchip with the colloid was then immediately covered with the second microchip. It has been reported that the diffusion of nanoparticles within the liquid cell enclosure is orders of magnitude slower than what would be expected for Brownian motion [4]. This enabled us to observe the self-assembly processes and the individual movements of the clusters within the temporal capabilities of STEM. In this way, we acquired a set of time lapse STEM image series, and analyzed the trajectories, distances and rotational movements of individual AuNPs and clusters as function of time. We found that the dynamical movements of AuNPs and clusters were driven by repulsive and attractive electrostatic forces. Unlike the random interactions caused by collisions due to Brownian motion, the AuNPs and the beam induced cluster structures followed specific trajectories with periods of immobilization of random waiting times between surface displacements or assembly events. Additionally, we observed attractive van der Waals interactions even when the distance between the surfaces of the AuNPs was in the range of 20-100 nm, van der Waals interactions are typically only considered for short surface-to-surface distances $(<10 \mathrm{~nm})$.

In summary, by liquid-phase STEM we observed and studied the dynamics of AuNPs at the solid:liquid interface. The self-assembly of the AuNPs was triggered by the radiolysis reactions of the water due to the electron beam. We found that the dynamical behavior of the AuNPs and the beam induced cluster structures at the solid:liquid interface involved long-range attractive interactions 
with periods of immobilization. This finding would help us to improve our understanding of surface transport of nanoparticles at solid:liquid interfaces [7].

\section{References:}

[1] MJ Skaug, J Mabry and DK Schwartz, Phys Rev Lett 110 (2013), 256101.

[2] GA Rance et al, ACS Nano 4 (2010), p. 4920.

[3] JN Israelachvili in "Intermolecular and surface forces", (Academic Press, Waltham, 2011).

[4] A Verch, M Pfaff and N de Jonge, Langmuir 31 (2015), p. 6956.

[5] J Kim et al, Nat Comm 8 (2017), p. 761.

[6] SW Chee et al, J Phys Chem C 120 (2016), p. 20462.

[7] We thank Eduard Arzt for his support through INM.

A

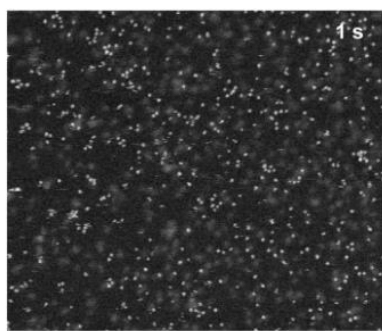

B

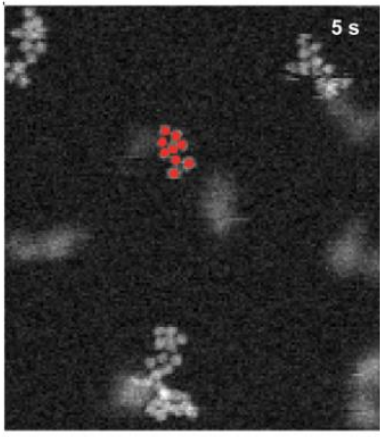

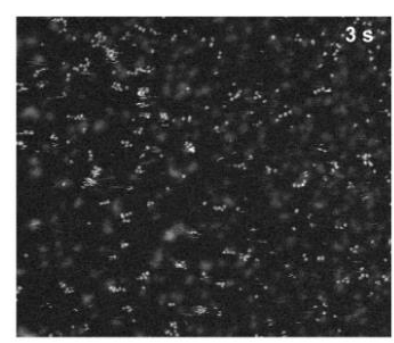

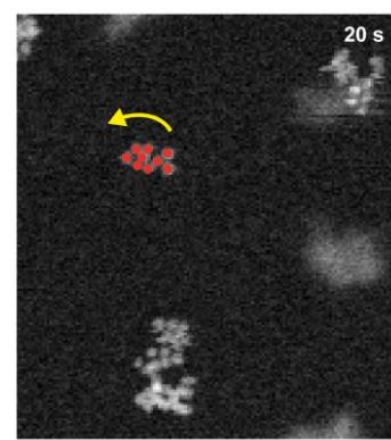

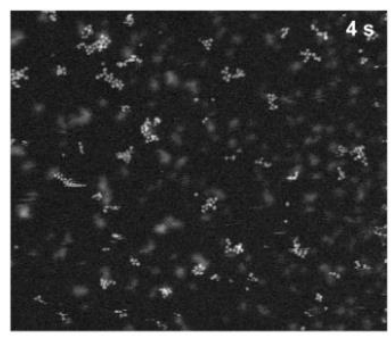
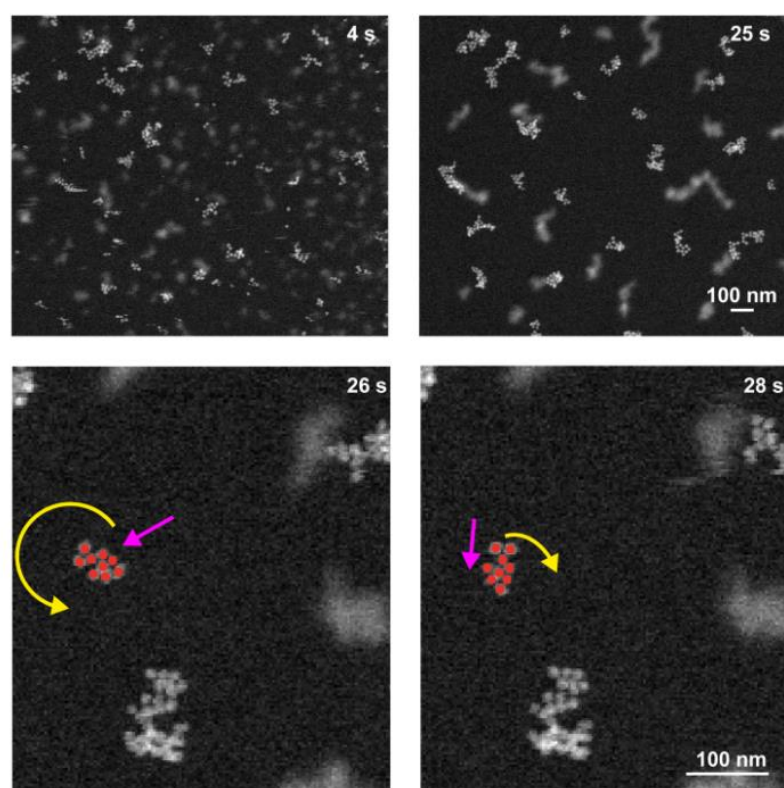

Figure 1. Dynamics of beam-induced gold nanoparticle (AuNP) aggregates. A. Stills from an annular dark field (ADF) STEM time-lapse series at different time points showing the formation of AuNP aggregates, most of the clustering process occurs during the first few seconds $(5 \mathrm{~s})$ of beam irradiation. B. Stills from an ADF STEM time-lapse series at different time points showing the dynamical behavior of an aggregate (marked in red), each frame corresponds to the time at which a change in the position or orientation of the aggregate was observed. The rotational movements and shifts of the aggregate were observed to occur in an intermittent manner. Electron dose: $1.1 \mathrm{e}-/ \mathrm{s} \AA^{2}$. 